\title{
Bidirectional WDM PON Enabled by Reflective ONUs and a Novel Overlapped-Subcarrier Multiplexing Technique
}

\author{
Ziad A. El-Sahn, Jonathan M. Buset, and David V. Plant \\ Photonics Systems Group, ECE Dept., McGill University, Montreal, QC H3A 2A7, Canada. \\ ziad_elsahn@ieee.org
}

\begin{abstract}
A symmetric WDM-PON architecture using an innovative overlapped-SCM scheme that maximizes the spectrum usage of a bandwidth-limited RSOA is demonstrated. Error-free operation is achieved using realistic launch powers without the need for DSP or FEC. OCIS codes: (060.2330) Fiber optics communications; (060.4250) Networks
\end{abstract}

\section{Introduction}

It has been widely acknowledged that the progression to wavelength-division multiplexing (WDM) is the natural choice for next generation passive optical network (PON) deployments in order to meet future bandwidth demands [1]. The use of colorless optical network unit (ONU) transmitters will be required to avoid the costs and inventory complexities associated with having distinct WDM transceivers [2-5]. Much of the current research has focused on the use of reflective semiconductor optical amplifiers (RSOAs) for this purpose, seeded with either continuous wave (CW) light from the optical line terminal (OLT) [3] or simply with the downstream signal [4]. However, in a single feeder PON, when the RSOA seed light has the same wavelength as that used for the downlink transmission, the system performance is severely degraded by Rayleigh backscattering [5]. Recently, subcarrier multiplexing (SCM) has been proposed to mitigate such degradation [5-8]. SCM may be used either for the uplink or the downlink, or for both transmissions as in [6]. Recently, bidirectional non-return-to-zero (NRZ) $1.25 \mathrm{~Gb} / \mathrm{s}$ uplink and differential phase shift keyed (DPSK) $2.5 \mathrm{~Gb} / \mathrm{s}$ SCM downlink were demonstrated [7]. However, doing the down-conversion only at the OLT makes the design of the ONU simpler by eliminating the need for a synchronized local oscillator (LO). As a result, this paper focuses only on the uplink SCM solutions, where up-conversion and down-conversion are carried at the ONU and OLT, respectively.

Due to the bandwidth limitation of the current generation RSOAs ( 2 GHz), most of the research on uplink SCM techniques has concentrated on asymmetrical and sub-gigabit per second bit rates. The subcarrier frequency and the bit rates are carefully chosen to separate both uplink and downlink enough to accommodate them within the modulation bandwidth of the RSOA. In [8] for example, a $622 \mathrm{Mb} / \mathrm{s}$ downlink was kept at baseband and a $100 \mathrm{Mb} / \mathrm{s}$ uplink was up-converted to a subcarrier frequency of $900 \mathrm{MHz}$. In this paper we introduce an innovative overlapped subcarrier multiplexing (O-SCM) technique that allows a certain overlap between uplink and downlink frequency spectra. Our inexpensive solution permits higher symmetrical bit rates by more efficiently utilizing the RSOA bandwidth compared to conventional SCM techniques, while also reducing the effects of Rayleigh backscattering. We successfully demonstrate a proof of concept experiment over a symmetrical $1.5 \mathrm{~Gb} / \mathrm{s}$ PON using a $1.5 \mathrm{GHz}$ subcarrier for the uplink. To the best of our knowledge, this is the maximum symmetrical bit rate reported for on-off keying (OOK) bidirectional SCM-WDM PONs using RSOAs and re-modulation, without forward error correcting (FEC) codes and without any electronic equalization. This realistic O-SCM approach also increases the link margin and can therefore be used to extend the PON reach and/or increase the capacity.

\section{Proposed WDM PON physical architecture and concept of overlapped-SCM}

The architecture of the O-SCM WDM PON in Fig. 1 employs existing infrastructure constraints to reduce potential deployment costs with a single feeder connecting the OLT to an arrayed waveguide grating (AWG)-based remote node (RN). Full duplex communication is achieved over the same feeder at the same wavelength using the proposed O-SCM technique (see insets in Fig. 1 showing the electrical RF spectrum for both uplink and downlink). The downlink is sent over the baseband, whereas the uplink is sent over an RF subcarrier using the proposed O-SCM. At the ONU, a fraction of the downlink power is used for detection by a $p-i-n$ photodetector and the remaining portion is used to seed the gain saturated RSOA for proper re-modulation. The splitting ratio of the ONU $1 \times 2$ coupler must therefore be carefully selected to maximize the power efficiency of the system. Moreover, the extinction ratio (ER) of the uplink is set higher than that of the downlink to facilitate data erasure and re-modulation [4].

The concept of the O-SCM is illustrated in Fig. 2(a) and (b) at $1 \mathrm{~Gb} / \mathrm{s}$, where part of the uplink spectrum overlaps with the residual downlink that is highly suppressed by the high pass filtering effect of the saturated RSOA. After down-conversion at the OLT, the uplink is recovered because the residual downlink only acts as an additive 


\section{OMP7.pdf}

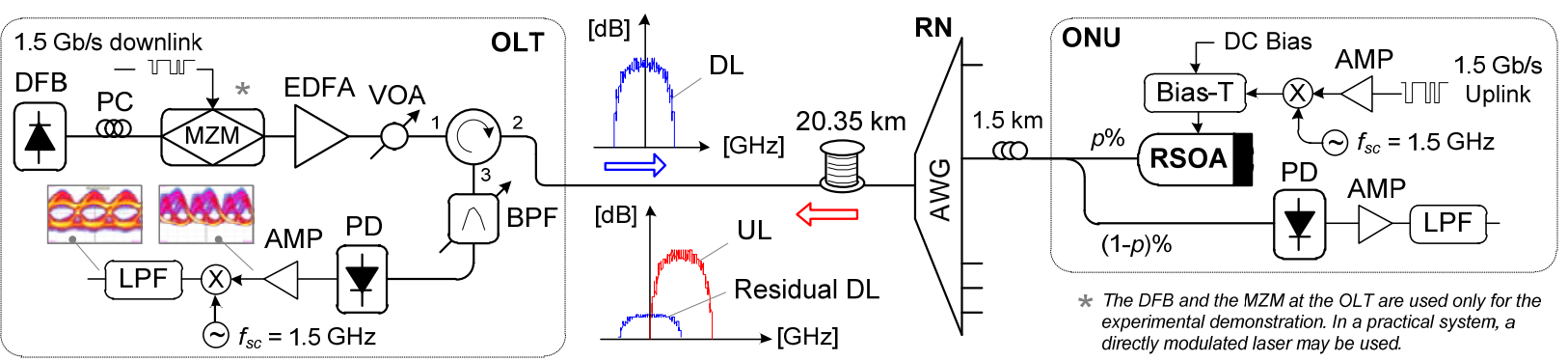

Fig. 1. Proposed WDM PON architecture with overlapped-SCM. (AMP: amplifier, BPF: 50 GHz band pass filter, DC: direct current, DFB: distributed feedback, DL: downlink, EDFA: erbium-doped fiber amplifier, $f_{s c}$ : subcarrier frequency, LPF: low pass filter, MZM: Mach-Zehnder modulator, PD: photodiode, PC: polarization controller, RF: radio frequency, UL: uplink, VOA: variable optical attenuator).

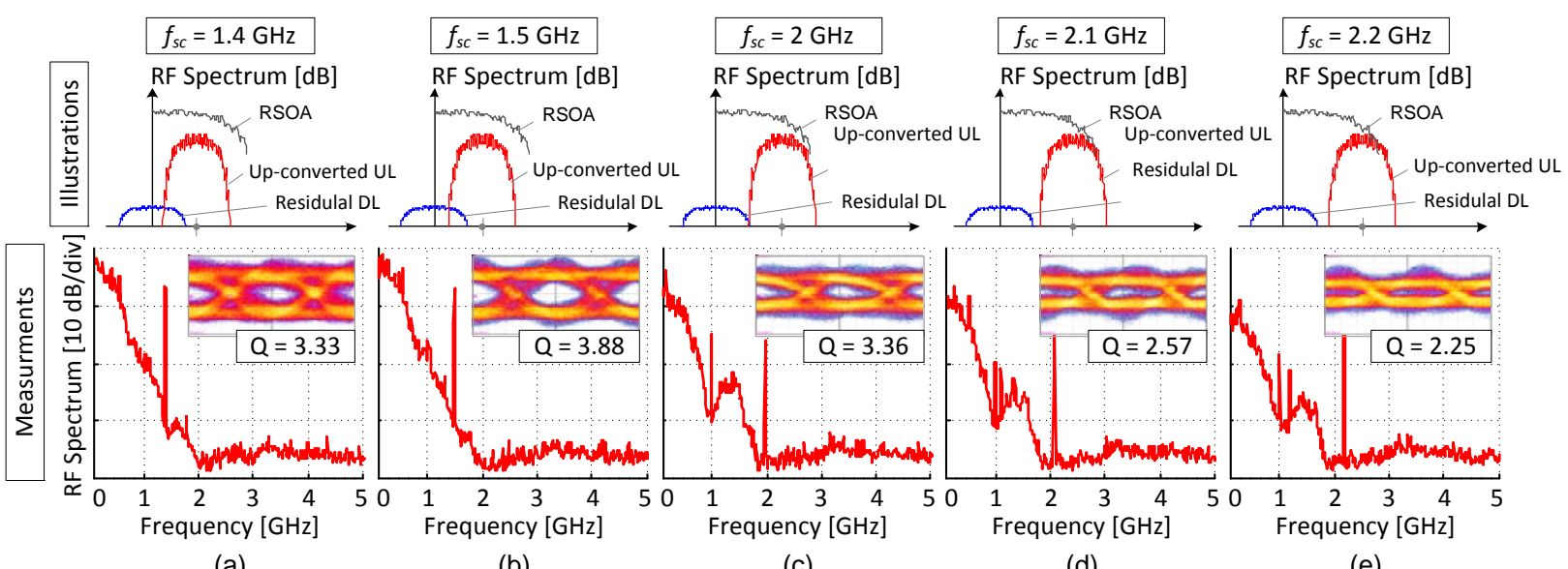

(a)

(b)

(c)

(d)

(e)

Fig. 2. Concept of the overlapped-SCM for $1 \mathrm{~Gb} / \mathrm{s}$ bit rate and different subcarrier frequencies (top), measured RF spectrum after downconversion (bottom): the spikes are due to the local oscillator leakage caused by the poor isolation of the RF mixers used.

high frequency noise that is suppressed by a low pass filter. This is illustrated in the lower subplots where the RF spectra, eye diagrams and Q factors are shown. For comparison purposes a more conventional SCM implementation is also shown in Fig. 2(c), (d) and (e). For a certain bit rate, the performance of the O-SCM technique is bounded on one side by the percentage of overlap and the RF mixer specifications, and by the modulation bandwidth of the RSOA on the other side. This explains why the best performance is achieved for the O-SCM when $f_{s c}=1.5 \mathrm{GHz}$. Moving to a higher subcarrier, as in Fig. 2(e), the performance is limited by the bandwidth of the RSOA (around $2 \mathrm{GHz}$ for the CIP: SOA-RL-OEC-1550), whereas moving to a lower subcarrier frequency degrades the performance due to the overlap and the mixers (Mini-Circuits: ZX05-C42MH). As the RSOA is more saturated, the downlink modulation will be highly suppressed allowing a 100\% O-SCM where $f_{s c}$ is equal to the bit rate.

This O-SCM scheme is therefore best targeted for next generation PONs as it efficiently uses the limited modulation bandwidth of the RSOA while reducing the effect of Rayleigh backscattering. Furthermore, the design of the ONU is inexpensive and simple enough to make our solution attractive for network operators.

\section{Experimental results and discussion}

The experimental setup for the O-SCM WDM PON is shown in Fig. 1. At the OLT side, a $1550.08 \mathrm{~nm}$ DFB laser is externally modulated at $1.5 \mathrm{~Gb} / \mathrm{s}$ with a $2^{15}-1 \mathrm{NRZ}$ pseudo random binary sequence (PRBS). The biasing of the Mach-Zehnder modulator is adjusted so that the ER is less than $2.5 \mathrm{~dB}$. An EDFA is then used with a VOA to control the launched power for the bit error rate (BER) measurements, but not necessary for system implementation where an electro-absorption modulated laser may replace the DFB and the MZM. At the ONU side, the RSOA is directly modulated at $1.5 \mathrm{~Gb} / \mathrm{s}$ with a $2^{23}-1 \mathrm{NRZ}$ up-converted $\left(f_{s c}=1.5 \mathrm{GHz}\right) \mathrm{PRBS}$. An RF amplifier and a bias-T are used to drive the RSOA (3.35 V DC with a $4 \mathrm{~V}$ peak-to-peak PRBS) so that the ER is significantly higher than $2.5 \mathrm{~dB}$. A 90/10 coupler is used to tap $10 \%$ of the downlink for reception. The same PD, RF amplifier and LPF are used at both ONU and OLT for reception. For the optical distribution network (ODN), standard single mode fiber (SMF-28e+) $20.35 \mathrm{~km}$ feeder and $1.5 \mathrm{~km}$ distribution drop fiber, and a $100 \mathrm{GHz}$ AWG are used.

Fig. 3(a) shows the BER performance for both downlink and uplink versus the received power (incident power on the PD). Error-free operation is achieved for both downlink and uplink with O-SCM. The 6 dB power penalty at 


\section{OMP7.pdf}

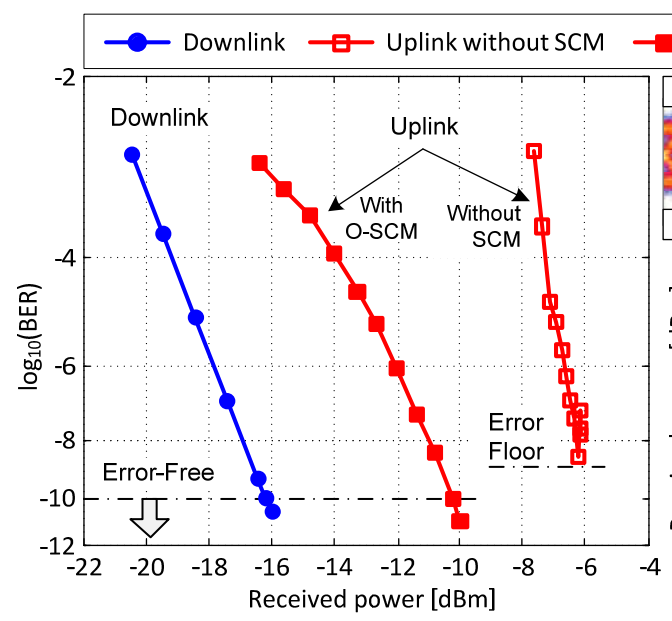

(a)

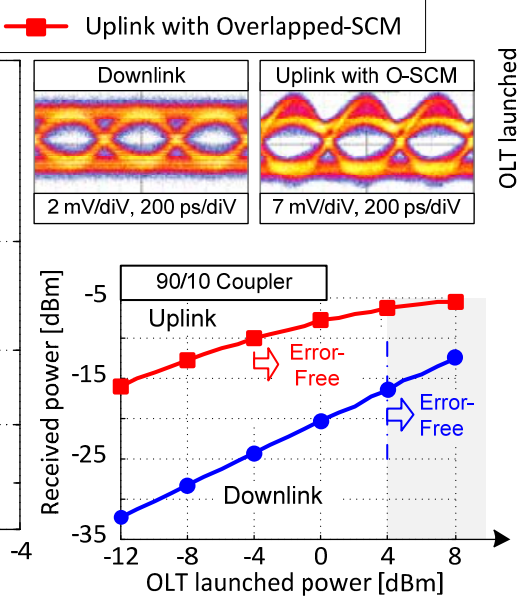

(b)

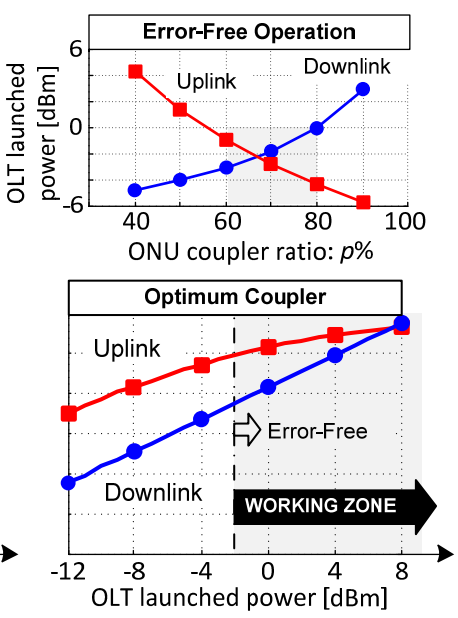

(c)

Fig. 3. (a) Measured BER versus the received power, (b) received power versus the OLT launched power for error-free operation, (c) the optimum ONU coupling ratio for error-free operation and the corresponding received power versus OLT launched power.

$10^{-10} \mathrm{BER}$ for the uplink is due to the additional losses within the ODN. We also show the performance of the uplink without SCM as a reference curve; the performance is highly degraded due to the Rayleigh backscattering and back reflections. The received power versus the OLT transmitted power is plotted in Fig. 3(b), indicating the error-free operating regions (in terms of transmitted power to achieve $10^{-10} \mathrm{BER}$ ) for both uplink and downlink. The working zone for both directions is represented by the shadowed region. Eye diagrams measured at $5 \mathrm{dBm}$ launched power are also shown. There is an $8 \mathrm{~dB}$ difference in launched power between uplink and downlink due to the use of a nonoptimized 90/10 ONU coupler which was the one available in our lab.

Based on our BER measurements and the link budget (insertion losses and the gain characteristics of the RSOA), we calculate in Fig. 3(c) the optimum ONU coupling ratio to minimize the OLT launched power while achieving error-free operation for both uplink and downlink. We also plot the received power versus the transmitted power showing the working zone. We found out the optimum configuration would be to send $-2 \mathrm{dBm}$ of power and to use a 70/30 coupler at the ONU side. Launching more power can help increase the reach and/or the capacity of the PON. A forward error correcting (FEC) with a BER threshold of $10^{-3}$ may also be used to provide around $4 \mathrm{~dB}$ and $6 \mathrm{~dB}$ of coding gain for the downlink and uplink, respectively. Combing our O-SCM with the latest digital signal processing (DSP) solutions for RSOA-based WDM PONs [9], the bit rate can easily be increased to $5 \mathrm{~Gb} / \mathrm{s}$ with no additional efforts. Possible upgrades to 10G PONs are likely with the continuous advances in DSP and equalization techniques from one side, and the development of new RSOAs with higher modulation speed from the other side.

\section{Conclusion}

We presented a novel bidirectional WDM PON based on an overlapped-SCM technique that exploits the bandwidth of the RSOA to its maximum compared to conventional SCM techniques. We successfully demonstrated a proof of concept experiment at $1.5 \mathrm{~Gb} / \mathrm{s}$, and optimized the OLT launched power based on the power budget. We showed that with only $-2 \mathrm{dBm}$ of OLT launched power; error-free performance can be achieved for a symmetrical $1.5 \mathrm{~Gb} / \mathrm{s}$ PON over a bidirectional $20.35 \mathrm{~km}$ feeder without dispersion compensation and without FEC.

\section{References}

[1] J. H. Lee, et al., "First commercial deployment of a colorless gigabit WDM/TDM hybrid PON system using remote protocol terminator," IEEE JLT 28, 344-351 (2010).

[2] F. Payoux, et al., "WDM-PON with colorless ONUs," in OFC, (Optical Society of America, 2007), Paper OTuG5.

[3] Z. Belfqih, et al., "Hybrid WDM-TDM passive optical network in burst mode configuration with RSOA," in OFC, (Optical Society of America, 2008), Paper JThA96.

[4] F. Payoux, et al., "Demonstration of a RSOA-based wavelength remodulation scheme in 1.25 Gbit/s bidirectional hybrid WDM-TDM PON," in OFC, (Optical Society of America, 2006), Paper OTuC4.

[5] C. Arellano, et al., "Reflections and multiple Rayleigh backscattering in WDM single-fiber loopback access networks," IEEE JLT 27, 12-18 (2009).

[6] C. Arellano, at al., "RSOA-based optical network units for WDM-PON," in OFC, (Optical Society of America, 2006 ), Paper OTuC1.

[7] J. M. Fàbrega, et al., "Demonstration of a full duplex PON featuring 2.5 Gbps sub carrier multiplexing downstream and 1.25 Gbps upstream with colourless ONU and simple optics,” in ECOC, (Institute of Electrical and Electronics Engineers, 2008), Paper We.1.F.6.

[8] J.-M. Kang, et al., "A novel hybrid WDM/SCM-PON sharing wavelength for up- and down-link using reflective semiconductor optical amplifier,” IEEE PTL 18, 502-504 (2006).

[9] K. Y. Cho, et al., “10-Gb/s operation of RSOA for WDM PON,” IEEE PTL 20, 1533-1535 (2010). 